\title{
Impacto da insuficiência renal crônica na qualidade de vida de crianças e adolescentes: revisão integrativa
}

\author{
Impact of chronic kidney disease on quality of life of children and adolescents: integrative review
}

Impacto de insuficiencia renal crónica en calidad de vida de niños y adolescentes: revisión integrativa

Isabella Schroeder Abreu ${ }^{1}$, Claudia Benedita dos Santos ${ }^{2}$

\footnotetext{
${ }^{1}$ Enfermeira, Doutora em Enfermagem em Saúde Pública. Professora Assistente da Universidade Estadual do Centro-Oeste. Guarapuava, PR, Brasil. E-mail: i_enf@yahoo.com.br.

${ }^{2}$ Matemática, Doutora em Estatística e Experimentação Agronômica. Professora Associada da Escola de Enfermagem de Ribeirão Preto da Universidade de São Paulo. Ribeirão Preto, SP, Brasil. E-mail: cbsantos@eerp.usp.br.
}

\section{RESUMO}

Pesquisas envolvendo mensuração do impacto da condição crônica sobre dimensões da qualidade de vida de crianças e adolescentes vêm aumentando de forma considerável. O objetivo desta revisão integrativa foi identificar, na literatura, aspectos emocionais, físicos e sociais relacionados à doença e seu tratamento, impactantes na qualidade de vida de crianças e adolescentes com insuficiência renal crônica, no período de 2000 a 2013. A identificação desses aspectos pode contribuir para a construção de instrumentos específicos válidos e fidedignos. Para seleção dos artigos, foram utilizadas bases de dados LILACS, SCOPUS e PUBMED. A amostra foi constituída de 17 artigos, descritos segundo objetivos, delineamento, método, resultados e nível de evidência científica. Resultados evidenciaram que ainda é difícil definição adequada da representação dos domínios e dimensões da qualidade de vida impactados pela condição crônica, relevantes para esse grupo, já identificados de forma consensual entre adultos.

Descritores: Qualidade de Vida; Insuficiência Renal Crônica; Criança; Adolescente.

\section{ABSTRACT}

Research that measures the increasing impact of chronic disease on the quality of life of children and adolescents. This integrative literature review sought to identify emotional, physical and social aspects related to disease and its treatment, which impact the quality of life of children and adolescents with chronic kidney disease, from 2000 to 2013. Identifying these aspects may help build specific valid and reliable instruments. Articles were selected from the databases: LILACS, SCOPUS and PUBMED. The sample was comprised of 17 articles, described according to objectives, design, method, results and level of scientific evidence. The results indicated that it is still difficult to adequately define the domains and dimensions of quality of life impacted by chronic disease, relevant to this group, consensually identified among adults.

Descriptors: Quality of Life; Renal Insufficiency, Chronic; Child; Adolescent.

\section{RESUMEN}

Las investigaciones enfocando la medición del impacto de la condición crónica en dimensiones de la calidad de vida de niños y adolescentes están en considerable auge. Esta revisión objetivó identificar en la literatura aspectos emocionales, físicos y sociales relativos a la enfermedad y su tratamiento, con impacto en calidad de vida de niños y adolescentes con insuficiencia renal crónica, entre 2000 y 2013. Identificar tales aspectos puede colaborar a la construcción de instrumentos específicos, válidos y fidedignos. Para seleccionar los artículos, fueron utilizadas las bases LILACS, SCOPUS y PUBMED. Muestra constituida por 17 artículos según objetivos, diseño, método, resultados y nivel de evidencia científica. Los resultados evidenciaron que aún es difícil definir adecuadamente la representación de los dominios y dimensiones de la calidad de vida impactadas por la condición crónica, relevantes para este grupo, ya identificadas de modo consensual entre adultos.

Descriptores: Calidad de Vida; Insuficiencia Renal Crónica; Niño; Adolescente. 


\section{INTRODUÇÃO}

A insuficiência renal crônica (IRC) é considerada uma condição sem alternativas de melhoras rápidas, de evolução progressiva, que causa problemas médicos, sociais e econômicos ${ }^{(1)}$. Constitui, na atualidade, um grande problema de saúde pública, considerando suas elevadas taxas de morbidade e mortalidade e, além disso, tem impacto negativo sobre a qualidade de vida (QV) dos indivíduos por ela acometidos.

QV é a "percepção do indivíduo de sua posição na vida no contexto da cultura e sistema de valores nos quais ele vive e em relação aos seus objetivos, expectativas, padrões e preocupações"(2). Entretanto, quando o termo qualidade de vida relacionada à saúde (QVRS) é utilizado, refere-se aos vários aspectos da vida de uma pessoa que são afetados por mudanças no seu estado de saúde e que são significativos para a sua $\mathrm{QV}^{(3)} \mathrm{O}$ diagnóstico de uma criança ou adolescente com IRC acarreta consequências negativas em sua QV/QVRS, bem como mudança na dinâmica familiar e alterações na rotina diária, decorrentes da demanda terapêutica, do controle clínico e das constantes hospitalizações.

QV tem se tornado importante critério na avaliação da efetividade de tratamentos e intervenções na área de saúde, sendo que as atenções começaram a se voltar, muito recentemente, para uma terapêutica visando à QV do paciente renal crônico como um fator relevante no cenário da terapêutica renal ${ }^{(4)}$. A assistência a crianças ou adolescentes com condições crônicas (CC) exige dos profissionais de saúde um conhecimento amplo e aprofundado para o desempenho de habilidades técnicas e científicas, com o objetivo de apreender a criança ou adolescente em sua subjetividade, bem como seu ambiente e sua família(5). Assim, o desenvolvimento de estudos sobre QV dessas pessoas enquanto um indicador a ser considerado no processo de avaliação mostra-se relevante.

O diagnóstico da IRC para crianças e adolescentes, em uma fase tão precoce de suas vidas, e as implicações impostas pela doença e tratamento em sua QV justificam o interesse em desenvolver uma revisão integrativa sobre o assunto para interpretação do conhecimento produzido na área e com o propósito de auxiliar no desenvolvimento de futuras investigações, que poderão favorecer melhorias em relação ao cuidado e à assistência prestada a esses pacientes, tendo em vista a redução do impacto negativo da condição crônica em sua QV.

O diagnóstico de uma criança ou adolescente com IRC acarreta consequências negativas em sua QV, bem como mudança na dinâmica familiar, com repercussões em várias esferas de sua vida, que impõem alterações na rotina diária e no convívio social. Essas modificações decorrem de necessidades dietéticas específicas, do compromisso com o tratamento dialítico, dos constantes procedimentos invasivos, do uso de medicamentos específicos e das hospitalizações em virtude de alterações clínicas frequentes ${ }^{(6)}$.

Os domínios físico, mental e social da QV podem ser negativamente impactados pela condição crônica, tendo em vista as repercussões causadas desde o momento em que essas crianças e adolescentes recebem a notícia do diagnóstico da doença, o estigma sofrido, a interrupção das atividades escolares em virtude do tratamento e a socialização prejudicada, entre outros.

Diante do exposto, o presente artigo teve como objetivo: identificar, na literatura, aspectos sociais, físicos e mentais associados à condição crônica e seu tratamento, impactantes na QV/QVRS de crianças e adolescentes com insuficiência renal crônica, no período de 2000 a 2013.

\section{MÉTODO}

Estudo utilizou o método de revisão integrativa, o qual permite reunir e sintetizar as evidências disponíveis sobre o tema investigado(7). Esse método de pesquisa caracteriza-se por seis fases distintas: identificação do tema ou questionamento da revisão integrativa; amostragem ou busca na literatura; categorização dos estudos; avaliação dos estudos incluídos na revisão integrativa; interpretação dos resultados; e síntese do conhecimento evidenciado nos artigos analisados ou apresentação da revisão integrativa ${ }^{(8-10)}$.

Foram percorridas as seguintes etapas: elaboração da questão de pesquisa; definição dos descritores para busca dos artigos, estabelecimento dos critérios de inclusão e exclusão; definição das informações a serem extraídas dos artigos selecionados; seleção dos artigos; análise e discussão dos resultados. A pergunta norteadora foi: Quais aspectos emocionais, físicos e sociais relacionados à doença e ao seu tratamento são impactantes na QV/QVRS de crianças e adolescentes com insuficiência renal crônica?

A identificação desses impactos, mencionados em trabalhos qualitativos ou quantitativos e relatados pela própria criança ou adolescente, responsáveis ou cuidadores, especialistas na temática QV/QVRS e/ou especialistas na área da saúde, pode contribuir para a construção de instrumentos específicos, válidos e 
fidedignos, que poderão ser utilizados para mensuração da qualidade de vida nessa população.

A revisão foi realizada utilizando-se as seguintes bases de dados: Base de dados da Literatura LatinoAmericana em Ciências da Saúde (LILACS), SCOPUS e Base de dados da Biblioteca Nacional de Medicina dos Estados Unidos (PUBMED). O acesso eletrônico à base LILACS foi realizado por meio da Biblioteca Virtual em Saúde (BVS); a SCOPUS foi acessada pelo Sistema Integrado de Bibliotecas Integradas (SIBI) da Universidade de São Paulo e a base PUBMED foi consultada por meio de endereço eletrônico.

Os critérios de inclusão foram: artigos publicados em português e inglês, no período de 2000 a 2013, cujos conteúdos abordassem o tema investigado, com resumos disponíveis e indexados nas bases anteriormente citadas. Foram excluídas as revisões bibliográficas, teses e dissertações. Para a busca nas bases de dados foram utilizados os seguintes descritores identificados nos Descritores em Ciência da Saúde (DECs): qualidade de vida (quality of life), criança (child) e insuficiência renal crônica (chronic renal insufficiency); posteriormente, o descritor criança foi substituído por adolescente (adolescent).

Para a seleção dos artigos, procedeu-se à leitura minuciosa dos títulos e dos resumos, atentando para sua relação com a questão norteadora e aos critérios de inclusão adotados. Para a análise das publicações, elaborou-se um roteiro com os seguintes dados: autores, ano e país de publicação, método, temática e resultados. Os artigos foram analisados individualmente pela pesquisadora principal.

Em relação ao nível de evidência (NE), os estudos foram classificados em forte (nível I e II), moderada (nível III, IV e V) e fraca (nível VI e VII) ${ }^{(11-12)}$. Vale salientar que na seleção dos artigos, em relação ao $\mathrm{NE}$, foram mantidos também os estudos qualitativos, pois a identificação dos impactos evidenciados por estudos qualitativos ou quantitativos pode contribuir para a construção de instrumentos específicos válidos e fidedignos que poderão ser utilizados para mensuração da qualidade de vida nessa população.

\section{RESULTADOS}

Na busca, utilizando-se o banco de dados LILACS, foi encontrado um total de 15 artigos, dos quais quatro foram selecionados. Na base SCOPUS, dos cinco artigos encontrados, foram escolhidos dois e, na base de dados PUBMED, foram selecionados 11 artigos dentre os 269 localizados. Assim, ao final, 17 artigos compuseram a amostra. As publicações repetidas em mais de uma base foram descartadas. Os artigos selecionados encontramse representados no Quadro 1. 
Quadro 1: Artigos selecionados.

\begin{tabular}{|c|c|c|c|c|c|}
\hline Autores & Objetivos & Delineamento & Método & Resultados & NE \\
\hline $\begin{array}{l}\text { Aguiar LK, Gazzinelli } \\
\qquad \mathrm{A}^{(13)}\end{array}$ & $\begin{array}{c}\text { Avaliar a QV de adolescentes } \\
\text { portadores de IRC em tratamento } \\
\text { conservador. }\end{array}$ & $\begin{array}{l}\text { Estudo caso } \\
\text { controle }\end{array}$ & $\begin{array}{l}\text { Aplicação de questionário em } \\
27 \text { adolescentes com IRC e em } \\
60 \text { saudáveis. }\end{array}$ & $\begin{array}{l}\text { Interferem na QV: percepção de saúde, planos para } \\
\text { o futuro, internações, dieta e preocupação com a } \\
\text { saúde. }\end{array}$ & IV \\
\hline $\begin{array}{l}\text { Rubik J, Grenda R, } \\
\text { Jakubowska WA, } \\
\text { Dabrowska A }{ }^{(14)}\end{array}$ & $\begin{array}{l}\text { Avaliar a QV de pacientes } \\
\text { transplantados e em diálise. }\end{array}$ & Estudo Transversal & $\begin{array}{c}\text { Foram analisados e } \\
\text { comparados os domínios do } \\
\text { instrumento aplicado em } 129 \\
\text { sujeitos. }\end{array}$ & $\begin{array}{l}\text { O domínios físicos e sociais são menos impactantes } \\
\text { nos pacientes transplantados. Concluiu-se que a } \\
\text { autoavaliação da QV entre os pacientes } \\
\text { transplantados é melhor do que no grupo em diálise. }\end{array}$ & IV \\
\hline $\begin{array}{l}\text { Riaño-Galán I, Málaga } \\
\text { S, Rajmil L }{ }^{(15)}\end{array}$ & $\begin{array}{c}\text { Investigar QV de } \\
\text { crianças/adolescentes com IRC e } \\
\text { comparar com saudáveis. }\end{array}$ & Estudo transversal & $\begin{array}{l}\text { Foi aplicado o instrumento } \\
\text { CHIP-AE, espanhol, em } 81 \\
\text { sujeitos. }\end{array}$ & $\begin{array}{c}\text { O domínio físico sofreu mais impacto nos pacientes } \\
\text { transplantados, porém o grupo em diálise foi o que } \\
\text { obteve menores escores no CHIP-AE. }\end{array}$ & IV \\
\hline $\begin{array}{c}\text { Gerson A, Hwang W, } \\
\text { Fiorenza J, Barth K, } \\
\text { Kaskel F, Weiss L, } \\
\text { Zelikovsky N, Fivush } \\
\text { B, Furth } \text { S }^{(16)} \\
\end{array}$ & $\begin{array}{l}\text { Verificar a associação entre anemia } \\
\text { e QVRS em adolescentes com IRC. }\end{array}$ & $\begin{array}{l}\text { Estudo de coorte, } \\
\text { prospectivo }\end{array}$ & $\begin{array}{l}\text { Aplicou-se, aos pais de } 105 \\
\text { adolescentes, o instrumento } \\
\text { CHQPF50. }\end{array}$ & $\begin{array}{c}\text { Pacientes anêmicos relataram limitações no } \\
\text { funcionamento físico, atividades escolares e com } \\
\text { amigos como resultado da saúde física e relações } \\
\text { familiares. }\end{array}$ & IV \\
\hline $\begin{array}{l}\text { Kul M, Cengel KE, } \\
\text { Senses DG, Bilginer Y, } \\
\text { Uluç S, Baykan H. }{ }^{(17)}\end{array}$ & $\begin{array}{l}\text { Comparar a QV de crianças e } \\
\text { adolescentes em vários estágios de } \\
\text { IRC, em diferentes modalidades de } \\
\text { tratamento com crianças e } \\
\text { adolescentes sadios. }\end{array}$ & Estudo transversal & $\begin{array}{l}\text { Foi aplicado o instrumento } \\
\text { Pediatric QoL Inventory TM, } \\
\text { em } 18 \text { pacientes } \\
\text { transplantados, } 21 \text { em dialise e } \\
\quad 37 \text { saudáveis. } \\
\end{array}$ & $\begin{array}{l}\text { Os domínios físico e psicossocial foram os que mais } \\
\text { impactaram a QV das crianças e adolescentes com } \\
\text { IRC. }\end{array}$ & IV \\
\hline \multirow{2}{*}{$\begin{array}{l}\text { Dodson JL, Diener- } \\
\text { WestM, Gerson AC, } \\
\text { Kaskel FJ, Furth SL }{ }^{(18)}\end{array}$} & \multirow{2}{*}{$\begin{array}{l}\text { Avaliar a QVRS de adolescentes } \\
\text { com IRC por DUC e comparar com } \\
\text { os pacientes em tratamento } \\
\text { conservador e população normal. }\end{array}$} & \multirow{2}{*}{$\begin{array}{l}\text { Estudo longitudinal } \\
\text { multicêntrico }\end{array}$} & \multirow{2}{*}{$\begin{array}{l}\text { Aplicação do instrumento } \\
\text { CHIP-AE, em } 113 \text { pacientes } \\
\text { com IRC. }\end{array}$} & $\begin{array}{l}\text { Os aspectos mais impactantes neste estudo foram:as } \\
\text { desordens causadas pela doença e os riscos fisicos. }\end{array}$ & \multirow[b]{2}{*}{ IV } \\
\hline & & & & $\begin{array}{l}\text { Os adolescentes com IRC por DUC obtiveram escore } \\
\text { estatisticamente maior do que os com IRC por outras } \\
\text { causas. }\end{array}$ & \\
\hline Sundaram S, cols ${ }^{(19)}$ & $\begin{array}{l}\text { Avaliar a QVRS de adolescentes pós } \\
\text { Tx de rim e fígado comparando com } \\
\text { uma população saudável. }\end{array}$ & Estudo Transversal & $\begin{array}{l}\text { Duas coortes de pacientes } \\
\text { foram recrutadas, sendo } \\
\text { aplicados os instrumentos } \\
\text { CHQ-CF } 87 \text { e o CHQ-PF } 50 \text {. }\end{array}$ & $\begin{array}{l}\text { Na percepção dos adolescentes, os aspectos físicos e } \\
\text { emocionais foram menos impactados. Os cuidadores } \\
\text { relataram menor saúde física e geral e semelhante } \\
\text { saúde psicológica a uma população normal. }\end{array}$ & IV \\
\hline \multirow{2}{*}{$\begin{array}{l}\text { Dodson Jl, Gerson AC, } \\
\text { Hsiao CJ, Kaskel FJ, } \\
\text { Weiss RA, Furth SL }{ }^{(20)}\end{array}$} & \multirow{2}{*}{$\begin{array}{l}\text { Avaliar a QVRS de adolescentes } \\
\text { com IRC por anomalias urológicas. }\end{array}$} & \multirow{2}{*}{$\begin{array}{l}\text { Estudo de coorte } \\
\text { prospectivo } \\
\text { multicêntrico }\end{array}$} & \multirow{2}{*}{$\begin{array}{l}\text { O CHQPF }-50 \text { foi aplicado em } \\
92 \text { pais dos adolescentes. }\end{array}$} & Neste estudo, o domínio físico sofreu mais impacto. & \\
\hline & & & & $\begin{array}{l}\text { Adolescentes com anomalias urológicas obtiveram } \\
\text { piores escores no domínio aspectos físicos. }\end{array}$ & IV \\
\hline $\begin{array}{l}\text { Goldstein SL, Rosburg } \\
\text { NM, Warady BA, } \\
\text { Seikaly M, Mc Donald } \\
\text { R, Limbers C, Varni } \\
\text { JW }^{(21)} \\
\end{array}$ & $\begin{array}{c}\text { Avaliar a QVRS de crianças com } \\
\text { IRC, em diferentes modalidades de } \\
\text { tratamento, utilizando o PedsQL } 3,0 \\
\text { ESRD. }\end{array}$ & Estudo transversal & $\begin{array}{l}\text { O instrumento foi autoaplicado } \\
\quad \text { para as crianças e } \\
\text { adolescentes e seus pais, em } 4 \\
\text { centros de nefrologia pediátrica } \\
\text { nos EUA. }\end{array}$ & $\begin{array}{c}\text { Pais de crianças/adolescentes transplantados } \\
\text { relataram melhor QVRS em relação aos em diálise, } \\
\text { exceto na Escala de Aparência Física percebida. Entre } \\
\text { as crianças, não houve diferenças estatisticamente } \\
\text { significativas, }\end{array}$ & IV \\
\hline
\end{tabular}




\begin{tabular}{|c|c|c|c|c|c|}
\hline Autores & Objetivos & Delineamento & Método & Resultados & NE \\
\hline \multirow{2}{*}{$\begin{array}{l}\text { Fernandez EA, } \\
\text { Izquierdo GE, Luque } \\
\text { PA, Garrido } \mathrm{CE}^{(22)}\end{array}$} & \multirow{2}{*}{$\begin{array}{l}\text { Avaliar como as crianças com IRC e } \\
\text { seus pais percebem sua saúde por } \\
\text { meio da mensuração da QVRS. } \\
\text { Avaliar os domínios da QVRS mais } \\
\text { afetados. }\end{array}$} & \multirow{2}{*}{ Estudo transversal } & \multirow{2}{*}{$\begin{array}{l}\text { Aplicado instrumento } \\
\text { Tecavner, em } 71 \text { crianças com } \\
\text { IRC e em seus pais. }\end{array}$} & $\begin{array}{l}\text { Os aspectos mais impactantes da QV foram os físicos } \\
\text { e sociais, sendo mais afetadas as atividades físicas e } \\
\text { escolares. }\end{array}$ & \multirow{2}{*}{ IV } \\
\hline & & & & $\begin{array}{c}\text { Crianças em HD referiram pior QVRS. Melhor QVRS } \\
\text { foi observada nos que se encontram em tratamento } \\
\text { conservador. }\end{array}$ & \\
\hline $\begin{array}{c}\text { Park KS, Cho MH, Ha } \\
\text { IS, Kang HG, Cheong } \\
\text { HI, Park YS, Lee YJ, } \\
\text { Lee JH, Cho } \mathrm{HY}^{(23)}\end{array}$ & $\begin{array}{l}\text { Validar a versão coreana do } \\
\text { PedsQoL 3.0 ESRD, comparando } \\
\text { com os PedsQL 4.0. }\end{array}$ & Estudo transversal & $\begin{array}{l}\text { Aplicou-se o PedsQol em } 92 \\
\text { pacientes pediátricos com IRC, } \\
\text { com idade entre } 2 \text { e } 18 \text { anos, e } \\
\text { em seus respectivos pais. }\end{array}$ & $\begin{array}{l}\text { O PedsQL } 3.0 \text { é um instrumento válido e confiável } \\
\text { para a avaliação de qualidade de vida de crianças } \\
\text { coreanas. }\end{array}$ & IV \\
\hline $\begin{array}{l}\text { Goldstein SL, Graham } \\
\text { N, Warady BA, } \\
\text { Seikaly M, Mc Donald } \\
\text { R, Burwinkle TM, } \\
\text { Limbers CA, Varni } \\
\text { JW }^{(24)}\end{array}$ & $\begin{array}{l}\text { Investigar a viabilidade e a validade } \\
\text { do PedsQL 3,0 DRT e do PedsQL 4,0 } \\
\text { Genérico. }\end{array}$ & Estudo Transversal & $\begin{array}{l}\text { Aplicação do PedsQL-ESRD e } \\
\text { do PedsQol } 4.0 \text { para avaliar a } \\
\text { QVRS de crianças com IRC, } \\
\text { comparando com crianças } \\
\text { saudáveis. }\end{array}$ & $\begin{array}{c}\text { A consistência interna para o PedsQL } 4,0 \text { e do } \\
\text { PedsQL } 3,0 \text { foi aceitável tanto para a versão self } \\
\text { quanto para a versão Proxy, com exceção de } 1 \text { relato } \\
\text { Proxy e } 3 \text { crianças, no módulo ESRD. }\end{array}$ & VI \\
\hline Falger J, et al. ${ }^{(25)}$ & $\begin{array}{l}\text { Avaliar a QV e o ajuste psicossocial } \\
\text { (PA) de crianças pós Tx renal. }\end{array}$ & Estudo Transversal & $\begin{array}{c}\text { Foram aplicados oS } \\
\text { instrumentos TNOAZL e } \\
\text { TACQOL para avaliar a QV e, } \\
\text { para avaliação do PA, aplicou- } \\
\text { se o instrumento CBCL em } 37 \\
\text { crianças e seus pais. }\end{array}$ & $\begin{array}{l}\text { Queixa física significativamente melhor e } \\
\text { funcionamento emocional positivo prejudicado em } \\
\text { relação aos controles sadios. Pais classificaram como } \\
\text { prejudicados: funcionamento motor, autonomia, } \\
\text { cognição, emoções positivas e PA em relação ao } \\
\text { tempo de diálise na comparação com crianças sadias. }\end{array}$ & VI \\
\hline $\begin{array}{l}\text { Vieira SS, Dupas G, } \\
\text { Ferreira NMLA }{ }^{(26)}\end{array}$ & $\begin{array}{l}\text { Compreender a vivência da criança } \\
\text { com IRC e seu significado. }\end{array}$ & $\begin{array}{l}\text { Descritivo, de } \\
\text { caráter qualitativo }\end{array}$ & $\begin{array}{l}\text { Utilizou o método Teoria } \\
\text { Fundamentada nos Dados. }\end{array}$ & $\begin{array}{l}\text { Emergiram oito categorias conceituais: descobrindo- } \\
\text { se doente, sofrendo mudanças no dia-dia, } \\
\text { prejudicando outrem, sofrendo com outros } \\
\text { problemas, procurando acostumar-se, igualando-se } \\
\text { às demais crianças, não sendo forte o bastante e } \\
\text { projetando o futuro. }\end{array}$ & VI \\
\hline \multirow{2}{*}{$\begin{array}{l}\text { Eijserman RM, } \\
\text { Creemers DG, Helders } \\
\text { PJ, Scroder } \mathrm{CH}^{(27)}\end{array}$} & \multirow{2}{*}{$\begin{array}{l}\text { Descrever as habilidades motoras, a } \\
\text { tolerância ao exercício e a QVRS em } \\
\text { crianças com IRC. }\end{array}$} & \multirow[b]{2}{*}{ Estudo transversal } & \multirow{2}{*}{$\begin{array}{l}\text { Estudo realizado com } 10 \\
\text { crianças com IRC em } \\
\text { hemodiálise. }\end{array}$} & Maior impacto da QV no domínio físico. & \multirow[b]{2}{*}{ VI } \\
\hline & & & & $\begin{array}{c}\text { A habilidade motora foi reduzida em funções motoras } \\
\text { brutas. A tolerância ao exercício foi restrita e os } \\
\text { pacientes em diálise relataram boa QVRS. }\end{array}$ & \\
\hline $\begin{array}{l}\text { Frota MA, Martins MC } \\
\text { Vasconcelos VM, }\end{array}$ & \multirow{2}{*}{$\begin{array}{c}\text { Avaliar a QV de crianças com IRC e } \\
\text { identificar os domínios mais } \\
\text { relevantes. }\end{array}$} & \multirow{2}{*}{$\begin{array}{l}\text { Descritivo, com } \\
\text { abordagem } \\
\text { quantitativa e } \\
\text { qualitativa. }\end{array}$} & \multirow{2}{*}{$\begin{array}{l}\text { Foi aplicado o instrumento } \\
\text { AUQEI em } 13 \text { crianças, no } \\
\text { período de janeiro a abril de } \\
2008 .\end{array}$} & \multirow{2}{*}{$\begin{array}{c}38,46 \% \text { têm a QV prejudicada, sendo lazer e família } \\
\text { com impacto menor e autonomia com maior } \\
\text { comprometimento. }\end{array}$} & \multirow{2}{*}{ VI } \\
\hline $\begin{array}{l}\text { Machado JC, Landin } \\
\text { FLP(28) }^{(28)}\end{array}$ & & & & & \\
\hline Abrahão SS et al. ${ }^{(29)}$ & $\begin{array}{c}\text { Descrever os relatos de cuidadores } \\
\text { sobre reações e dificuldades da } \\
\text { família da criança/adolescente com } \\
\text { IRC. }\end{array}$ & $\begin{array}{l}\text { Descritivo, de } \\
\text { caráter qualitativo. }\end{array}$ & $\begin{array}{l}\text { Dados obtidos por meio de } \\
\text { entrevistas, consultas e visita } \\
\text { domiciliar. }\end{array}$ & $\begin{array}{l}\text { As principais queixas foram: limitações trazidas pelo } \\
\text { tratamento, tanto para o paciente quanto para os } \\
\text { pais, sendo que os aspectos físicos, sociais e } \\
\text { emocionais impactaram a QV do grupo estudado. }\end{array}$ & VI \\
\hline
\end{tabular}


As publicações internacionais referiam-se, em sua maioria, a periódicos específicos de nefrologia pediátrica, além de periódicos também da área de nefrologia que abordavam temas específicos como: transplante renal, diálise peritoneal, doenças renais, entre outros. Entre os artigos, 14 tinham abordagem quantitativa, sendo quatro longitudinais (um estudo caso-controle, dois estudos de coorte prospectivos e um multicêntrico) e 10 transversais, dois estudos com abordagem qualitativa e um envolvendo delineamentos qualitativo e quantitativo.

Em relação aos objetivos, 10 artigos referiram-se especificamente à avaliação de $\mathrm{QV}^{(13-15,18-20,22,24-25,28)}$, sendo que os demais apresentaram objetivos relacionados às repercussões emocionais e comprometimento da QV/QVRS que possam influenciar no prognóstico dessas crianças e adolescentes; a compreensão da vivência e dos significados que a criança e seus familiares atribuem à IRC e seu tratamento e as implicações da IRC para o paciente pediátrico ${ }^{(26)}$; investigar a viabilidade, confiabilidade e a validade do instrumento PedsQoL 3,0-Módulo ESRD ${ }^{(21)}$; validar a versão coreana do PedsQol 3,0-Módulo ESRD(23), descrever as habilidades motoras, a tolerância ao exercício e a QVRS de crianças com IRC ${ }^{(27)}$, verificar a associação entre anemia e QVRS em adolescentes com IRC ${ }^{(16)}$, descrever e discutir relatos de cuidadores sobre as reações e dificuldades vivenciadas pelos familiares e por crianças e adolescentes com IRC ${ }^{(29)}$.

Dentre os artigos selecionados, 12 (70,5\%) utilizaram instrumentos para avaliar, investigar, descrever ou comparar a QV/QVRS de crianças e adolescentes com IRC e seus respectivos pais ou cuidadores $^{(15-25,28)}$, sendo que dois estudos não mencionaram o nome dos questionários aplicados ${ }^{(13-14)}$. Os instrumentos utilizados nos estudos foram: Autoquestionnaire Qualité de Vie Enfant Imagé (AUQEI), The Child Health and IIness Profile - Adolescente Edition (CHIP-AE), Teste de Calidad de Vida en Niños con Enfermedad Renal (TECAVNER), Peds QL 3,0 End Stage Renal Disease (ESRD), Child Health and Ilness Profile Parent Form 50 (CHQ-PF 50), Child Health and Ilness Profile - Children Form 87 (CHQ-PF 87), TNO-AZL Questionnaires for Children's Health-Related Quality of Life (TACQOL), Kinder Lebensqualität Fragebogen, Impact on Family Scale- German Adaptation (IFS), Social Orientation of Parents of Handicapped Children Questionnaire SOEBEK (FKV), Freiburg Questionnaire of Coping With Wilness e UIm Quality of Life Inventory for Parents (ULQIE).
Desses, apenas dois são específicos para avaliar QV/QVRS de crianças e adolescentes com IRC: o TECAVNER, versão espanhola, desenvolvido com base no questionário de QV para adultos com IRC (KDQOL SFTM) ${ }^{(22)}$ e no questionário de QV para crianças com epilepsia (CAVE), e o Peds QL 3,0 End Stage Renal Disease (ESRD), validado em $2008^{(21)}$.

Em relação ao impacto, principalmente da IRC e de seu tratamento nos domínios ou dimensões da QV/QVRS das crianças e adolescentes, foram encontrados 11 estudos, sendo oito com nível de evidência IV e três VI.

Três estudos mostraram que este grupo apresenta comprometimento de sua autonomia e que as principais limitações estão relacionadas ao próprio tratamento, influenciando negativamente em relação a aspectos físicos e ao comprometimento das atividades escolares e familiares $^{(16,22,29)}$.

Dois estudos revelaram que os domínios ou dimensões mais impactados foram: lazer, família e autonomia, sendo a última a mais comprometida ${ }^{(25,28)}$.

Dois estudos evidenciaram que crianças e adolescentes com IRC que realizaram transplante renal e seus respectivos pais/cuidadores relataram uma melhor QV/QVRS do que aqueles submetidos a tratamento dialítico ${ }^{(14,19)}$.

Os dois estudos que comparam a QV/QVRS entre grupos de crianças e adolescentes com IRC em diferentes modalidades de tratamento ${ }^{(17,24)}$ mostraram que crianças e adolescentes em hemodiálise apresentam maior comprometimento da QV/QVRS quando comparados com aqueles submetidos a tratamento conservador, transplantados ou em diálise peritoneal. Embora não discutido pelos autores do estudo, esse resultado pode estar associado principalmente ao fato de que essas pessoas precisam se deslocar às unidades de diálise três vezes por semana, permanecendo em hemodiálise por um período de até 4 h00 por sessão, além das queixas que referem, relacionadas à dor no momento da punção da fístula arteriovenosa (FAV) e às complicações durante o tratamento.

\section{DISCUSSÃO}

A doença renal é considerada um grande problema de saúde pública em virtude de suas elevadas taxas de morbidade e mortalidade e, além disso, tem impacto negativo sobre a QV/QVRS. Estudos acerca da QV/QVRS de crianças e adolescentes tiveram início nos anos $80^{(30)}$; no entanto, quantificar QV/QVRS é difícil e considerado um desafio que incentiva o desenvolvimento de novas pesquisas. 
A melhoria da atenção médica resultou em um aumento da sobrevida de pacientes pediátricos com IRC, porém são inúmeras as repercussões clínicas e as consequências do tratamento ${ }^{(31)}$. O diagnóstico de uma criança ou adolescente com IRC acarreta consequências negativas em sua QV/QVRS, bem como mudança na dinâmica familiar e alterações na rotina diária, conforme evidenciado nos artigos encontrados. Essas mudanças estão relacionadas à reestruturação da alimentação, uso de medicamentos específicos e ao tratamento dialítico. Ocorrem, também, mudanças no orçamento familiar, limitando a vida social, sendo que as famílias, na maioria das vezes, não estão preparadas para compreender este diagnóstico.

Os estudos incluídos nesta revisão visaram identificar os principais aspectos impactantes nas dimensões social, física e mental da QV/QVRS de crianças e adolescentes com IRC, relacionados à sua doença e ao seu tratamento. Os artigos encontrados abordaram temas referentes à avaliação de QV/QVRS em crianças e adolescentes com IRC em tratamento, aos quais foram aplicados instrumentos genéricos $e$ específicos para avaliação da QV/QVRS, além de estudos qualitativos mostrando repercussões emocionais e o comprometimento da QV/QVRS dessa população que podem influenciar no seu prognóstico e controle clínico. Entre os estudos, sete (41\%) destacaram as dimensões da QV/QVRS negativamente impactadas pela condição crônica e tratamento.

Entretanto, 14 artigos mencionaram, com base em seus resultados, a importância da utilização e da elaboração de instrumentos específicos para avaliar a QV/QVRS dessa população, salientando que a mensuração da QV/QVRS é fundamental para o conhecimento do estado de saúde e bem-estar. Referiram, também, que crianças e adolescentes com IRC podem apresentar comprometimento da sua QV/QVRS e de sua saúde mental. É importante ressaltar que, no Brasil, encontramos apenas um instrumento, traduzido e adaptado culturalmente para a língua portuguesa, destinado à avaliação da QV/QVRS de crianças e adolescentes renais crônicos ${ }^{(6)}$.

Tais aspectos reforçam a relevância da mensuração da QV/QVRS em crianças e adolescentes e a dificuldade de se definir adequadamente a representação dos domínios e dimensões relevantes para esse grupo, já identificados de forma consensual entre adultos ${ }^{(32)}$.

Os resultados sinalizam para a importância de adequar os domínios e dimensões da QV/QVRS, de modo a refletirem, de modo fidedigno, o mundo da criança e do adolescente.

\section{CONCLUSÃO}

Apesar do crescimento das publicações sobre QV/QVRS de crianças e adolescentes com IRC, a temática ainda é pouco explorada. Acredita-se que esse crescimento venha ocorrendo principalmente em virtude de uma maior atenção, por parte dos profissionais de saúde, às doenças crônicas nas últimas décadas, o que decorre de seu impacto significativo na morbimortalidade da população mundial, em especial jovens em idade produtiva, crianças e adolescentes.

O enfoque da maioria dos estudos na avaliação da QV/QVRS de crianças e adolescentes com IRC deve-se principalmente ao seu crescimento e incidência nessa população e ao impacto negativo que acarreta nos domínios ou dimensões da QV/QVRS dessas pessoas. Assim, sua avaliação deve envolver especialistas, pais, responsáveis ou cuidadores e, sobretudo, as crianças e adolescentes com IRC, enquanto conhecedores de sua própria condição, de modo a oferecer medidas válidas e fidedignas para pesquisas em doença renal crônica, dada a carência, no Brasil, de tais instrumentos de avaliação.

A identificação de aspectos impactantes da QV/QVRS dessa população também permitirá aos profissionais de saúde repensar a assistência atualmente oferecida, com vistas a auxiliá-los e assim minimizar as consequências negativas decorrentes desse processo. Acredita-se que esse conhecimento contribuirá para que se adaptem às mudanças e criem suas próprias ferramentas internas de enfrentamento das limitações impostas pela condição crônica.

Considera-se que os objetivos deste estudo tenham sido alcançados e espera-se que os resultados de investigações tragam subsídios para a elaboração de instrumentos de mensuração da QV/QVRS de crianças e adolescentes com IRC, visando à melhoria da qualidade da assistência à saúde atualmente prestada a essas pessoas.

\section{REFERÊNCIAS}

1. Madeira EPQ, Lopes GS, Santos SFF. A investigação epidemiológica na prevenção da insuficiência renal terminal. Ênfase no estudo da agregação familiar. Medicina On line
[Internet]. 1998 [acesso em: $31 \mathrm{dez} 2014$ ];1(2). Disponível em: http://bit.ly/17YxuHv.

2. The WHOQOL Group. The development of the World Health Organization quality of life assessment instrument (the 
WHOQOL). In: Orley J, Kuyken W, editors. Quality of life assessment: international perspectives.Heidelberg: Springer Verlag; 1994. p. 41-60.

3. Cleary PD, Wilson PD, Fowler FJ. Health-related quality of life in HIV- Infected persons: a conceptual model. In: Dimsdale JE, Baum A. Quality of life in behavioral medicine research. New Jersey: Lawrence Erlbaum Associates; 1995. p. 191-204. 4. Suzuki K. Pesquisa sobre a qualidade de vida de pacientes de UTI: uma revisão de literatura [Dissertação]. São Paulo: Escola de Enfermagem/USP; 2002.

5. Rocha SMM, Lima RAG, Scochi CGS, Vendrúsculo DMS, Mello DF. Estudo da assistência integral à criança e ao adolescente através da pesquisa qualitativa. Rev Lat Am Enfermagem [Internet].1998 [acesso em: 31 dez 2014];6(5):5-15. Disponível em: http://dx.doi.org/10.1590/S0104-11691998000500002. 6. Lopes M, Koch VHK, Varni JW. Translation and cultural adaptation of Peds QL ${ }^{\mathrm{TM}}$ ESRD to Portuguese. J Bras Nefrol [Internet]. 2011 [acesso em: $31 \mathrm{dez} 2014$ ];33(4):448-56. Disponível em: http://dx.doi.org/10.1590/S010128002011000400010.

7. Pompeo DA, Rossi LA, Galvão CM. Revisão integrativa: etapa inicial do processo de validação de diagnóstico de enfermagem. Acta paul. enferm. [Internet]. 2009 [acesso em: $31 \mathrm{dez}$ 2014];22(4):1-6. Disponível em:

http://dx.doi.org/10.1590/S0103-21002009000400014.

8. Whittemore $\mathrm{R}$, Knafl $\mathrm{K}$. The integrative review: updated methodology. J Adv Nurs [Internet]. 2005 [acesso em: $31 \mathrm{dez}$ 2014];52(5):546-53. Disponível em: http://dx.doi.org/10.1111/j.1365-2648.2005.03621.x.

9. Broome MA. Integrative literature reviews for the development of concepts. In: Rodgers BL, Knafl KA.Concept development in nursing: foundations, techniques, and applications. Philadelphia: Saunders; 2000. p. 231-50.

10. Ganong LH. Integrative reviews of nursing research. Res Nurs Health [Internet]. 1987 [acesso em: 31 dez 2014];10(1):111. Disponível em: http://dx.doi.org/10.1002/nur.4770100103. 11. Santos DRF, Silva FBL, Saldanha EA, Lira ALBC, Vitor AF. Cuidados de enfermagem ao paciente em pós-operatório de prostatectomia: revisão integrativa. Rev. Eletr. Enf. [Internet]. 2012 [acesso em: 31 dez 2014];14(3): 690-701. Disponível em: http://dx.doi.org/10.5216/ree.v14i3.14980.

12. Melnyk BM, Fineout-Overholt E. Making the case for evidence-based practice. In: Melnyk BM, Fineout-Overholt E. Evidence based practice in nursing \& healthcare. A guide to best practice. Philadelphia: Lippincot Williams \& Wilkins; 2005. p. 324.

13. Aguiar LK, Gazzinelli A. Qualidade de vida de adolescentes portadores de insuficiência renal crônica. X Congresso Brasileiro de Enfermagem em Nefrologia, 2000, Natal, BR. Natal: SOBEN; 2000.

14. Rubik J, Grenda R, Jakubowska-Winecka A, Dabrowska A. [Quality of life in children and adolescent with end-stage renal disease treated with dialysis and kidney transplantation]. Pol Merkur Lekarski. 2000 Apr;8(46):280-1. [Article in Polish]. 15. Riaño-Galán I, Málaga S, Rajmil L, Ariceta G, Navarro M, Loris $C$ et al. Quality of life of adolescents with end-stage renal disease and kidney transplant. Pediatr Nephrol [Internet]. 2009 [acesso em: $31 \mathrm{dez} 2014] ; 24(8): 1561-8$. Disponível em: http://dx.doi.org/10.1007/s00467-009-1175-0.

16. Gerson A, Hwang W, Fiorenza J, Barth K, Kaskel F, Weiss L et al. Anemia and health-related quality of life in adolescents with chronic kidney disease. Am J Kidney Dis [Internet]. 2004 [acesso em: 31 dez 2014];44(6):1017-23. Disponível em: http://dx.doi.org/10.1053/j.ajkd.2004.08.024.

17. Kul M, Cengel Kültür $E$, Senses Dinç G, Bilginer $Y$, Uluç $S$, Baykan $\mathrm{H}$. Quality of life in children and adolescents with chronic kidney disease: a comparative study between different disease stages and treatment modalities. Turk J Pediatr.

2013;55(5):493-9.

18. Dodson JL, Diener-West M, Gerson AC, Kaskel FJ, Furth SL. An assessment of health related quality of life using the child health and illness profile-adolescent edition in adolescents with chronic kidney disease due to underlying urological disorders. J
Urol [Internet]. 2007 [acesso em: $31 \mathrm{dez} 2014$ ];178(2):660-5. Disponível em: http://dx.doi.org/10.1016/j.juro.2007.04.008. 19. Sundaram SS, Landgraf JM, Neighbors K, Cohn RA, Alonso EM. Adolescent health-related quality of life following liver and kidney transplantation. Am J Transplant [Internet]. 2007 [acesso em: 31 dez 2014];7(4):982-9. Disponível em:

http://dx.doi.org/10.1111/j.1600-6143.2006.01722.x. 20. Dodson JL, Gerson AC, Hsiao CJ, Kaskel FJ, Weiss RA, Furth $\mathrm{SL}$. Parent perspectives of health related quality of life in adolescents with chronic kidney disease due to underlying urological disorders: an assessment using the Child Health Questionnaire-Parent Form 50. J Urol [Internet]. 2008 [acesso em: 31 dez 2014];180(4 Suppl):1700-4. Disponível em: http://dx.doi.org/10.1016/j.juro.2008.03.117.

21. Goldstein SL, Graham N, Warady BA, Seikaly M, McDonald R, Burwinkle TM et al. Measuring health-related quality of life in children with ESRD: performance of the generic and ESRDspecific instrument of the Pediatric Quality of Life Inventory (PedsQL). Am J Kidney Dis [Internet]. 2008 [acesso em: $31 \mathrm{dez}$ 2014];51(2):285-97. Disponível em:

http://dx.doi.org/10.1053/j.ajkd.2007.09.021.

22. Aparicio López C, Fernández Escribano A, Luque de Pablos A, Isquierdo Garcia E, Garrido Cantanero G. Desarrollo de un cuestionario en español de medida de calidad de la vida en pacientes pediátricos con enfermedad renal crónica. Nefrologia [Internet]. 2010 [acesso em: $31 \mathrm{dez} 2014$ ];30(2):168-76. Disponível em:

http://dx.doi.org/10.3265/Nefrologia.pre2009.Dic.5684. 23. Park KS, Cho MH, Ha IS, Kang HG, Cheong HI, Park YS et al. Validity and reliability of the Korean version of the pediatric quality of life ESRD module. Health and Quality of Life Outcomes [Internet]. 2012 [acesso em: $31 \mathrm{dez} 2014$ ];10:59. Disponível em: http://dx.doi.org/10.1186/1477-7525-10-59.

24. Goldstein SL, Rosburg NM, Warady BA, Seikaly M, McDonald $R$, Limbers $C$ et al. Pediatric end stage renal disease healthrelated quality of life differs by modality: a PedsQL ESRD analysis. Pediatr Nephrol [Internet]. 2009 [acesso em: $31 \mathrm{dez}$ 2014];24(8):1553-60. Disponível em: http://dx.doi.org/10.1007/s00467-009-1174-1.

25. Falger J1, Landolt MA, Latal B, Rüth EM, Neuhaus TJ, Laube GF. Outcome after renal transplantation. Part II: quality of life and psychosocial adjustment. Pediatr Nephrol [Internet]. 2008 [acesso em: 31 dez 2014];23(8):1347-54. Disponível em: http://dx.doi.org/10.1007/s00467-008-0798-x. 26. Vieira SS, Dupas G, Ferreira NMLA. Doença renal crônica: conhecendo a experiência da criança. Esc. Anna Nery [Internet] 2009 [acesso em: $31 \mathrm{dez} 2014] ; 13(1): 74-83$. Disponível em: http://dx.doi.org/10.1590/S1414-81452009000100011. 27. Eijsermans RM, Creemers DG, Helders PJ, Schröder CH. Motor performance, exercise tolerance, and health-related quality of life in children on dialysis. Pediatr Nephrol [Internet]. 2004 [acesso em: $31 \mathrm{dez} 2014] ; 19(11): 1262-6$. Disponível em: http://dx.doi.org/10.1007/s00467-004-1583-0.

28. Frota MA, Machado JC, Martins MC, Vasconcelos VM, Landin FLP. Qualidade de vida da criança com insuficiência renal crônica. Esc. Anna Nery [Internet]. 2010 [acesso em: $31 \mathrm{dez}$ 2014];14(3):527-33. Disponível em: http://dx.doi.org/10.1590/S1414-81452010000300014. 29. Abrahão SS, Ricas J, Andrade DF, Pompeu FC, Chamahum L, Araújo TM et al. Dificuldades vivenciadas pela família e pela criança/adolescente com doença renal crônica. J Bras Nefrol [Internet]. 2010 [acesso em: $31 \mathrm{dez} 2014$ ];32(1):18-22. Disponível em: http://dx.doi.org/10.1590/S010128002010000100004.

30. Bullinger $M$, Ravens-sieberer $U$. Health related quality of life assessment in children: a review of the literature. Eur Rev Appl Psychol. 1995;(45):245-54.

31. Marciano RC, Soares CMB, Diniz JSS, Lima EM, Silva JMP, Canhestro MR et al. Transtornos mentais e qualidade de vida em crianças e adolescentes com doença renal crônica e em seus cuidadores. J Bras Nefrol [Internet]. 2010 [acesso em: $31 \mathrm{dez}$ 2014];2010;32(3):316-22. Disponível em: http://dx.doi.org/10.1590/S0101-28002010000300014. 
32. von Mackensen S, Bullinger M, HAEMO-QOL GROUP.

Development and testing of an instrument to assess the Quality

of Life of Children with Haemophilia in Europe (Haemo-QoL).

Haemophilia [Internet]. 2004 [acesso em: $31 \mathrm{dez} 2014$ ];10

Suppl 1:17-25. Disponível em: http://dx.doi.org/10.1111/j.1355-

0691.2004.00875.x.

Artigo recebido em 27/02/2013.

Aprovado para publicação em 02/06/2014.

Artigo publicado em 31/12/2014. 